\title{
Anlotinib Exerts Anti-Cancer Effects on KRAS-Mutated Lung Cancer Cell Through Suppressing the MEK/ERK Pathway
}

This article was published in the following Dove Press journal: Cancer Management and Research

\author{
Haoyue $\mathrm{Hu}$ (D) $^{\mathrm{I} *}$ \\ Yanyang Liu ${ }^{1, *}$ \\ Songtao $\operatorname{Tan}^{1, *}$ \\ Xiao Xiao Xie' \\ Jun $\mathrm{He}^{1,2}$ \\ Feng Luo' \\ Li Wang'
}

'Lung Cancer Center, Cancer Center, State Key Laboratory of Biotherapy, West China Hospital of Sichuan University, Chengdu, Sichuan, People's Republic of China; ${ }^{2}$ Department of Oncology, The Third Hospital of Mianyang (Sichuan Mental Health Center), Mianyang,

Sichuan, People's Republic of China

*These authors contributed equally to this work
Correspondence: Feng Luo; Li Wang Lung Cancer Center, Cancer Center, State Key Laboratory of Biotherapy, West China Hospital of Sichuan University, Chengdu, Sichuan, People's Republic of China

Email hxyyluofeng@sina.com; wangli37029192@sina.com
Background: With a high frequency of $30 \%$, KRAS mutations in patients with non-small cell lung cancer (NSCLC) often lead to their poor response to most anti-cancer therapies. As a multi-target tyrosine kinase inhibitor, Anlotinib shows clinical efficacy against several types of cancer. However, its effects on KRAS mutant NSCLC and the underlying molecular mechanisms remain unclear.

Materials and Methods: Cell counting Kit- 8 assay, colony formation assay, flow cytometry analysis, wound healing scratch assay, Transwell assay and xenograft mouse model were used to evaluate the anti-cancer effects of Anlotinib. The potential molecular mechanisms were determined by immunohistochemistry (IHC) and Western blotting.

Results: Anlotinib inhibited proliferation of KRAS mutant lung cancer cells and induced apoptosis in vitro. In addition, the migration and invasion abilities of these cells were also decreased after treatment with Anlotinib. It significantly suppressed tumor growth in vivo and prolonged the survival of the xenograft-bearing mice, which correlated to lower expression levels of Ki67 in the tumor tissues. Mechanistically, Anlotinib downregulated MEK and ERK as well as their phosphorylated forms in the KRAS mutant lung cancer cells.

Conclusion: Anlotinib inhibits the growth of KRAS mutant lung cancer cells partly via the suppression of the MEK/ERK pathway. Our findings provide novel insights into treating recalcitrant KRAS mutated NSCLC.

Keywords: Anlotinib, KRAS-mutated, lung cancer, signal pathways

\section{Introduction}

Lung cancer is a commonly diagnosed malignancy associated with high morbidity and mortality. Non-small cell lung cancer (NSCLC) accounts for $80 \%$ of all lung cancer cases. ${ }^{1}$ Cisplatin-based chemotherapy has been the mainstay of advanced NSCLC treatment, although the 5-year survival rate is less than $5 \%{ }^{2}$ With the advent of targeted therapy, the prognosis and survival of NSCLC patients harboring mutations such as EGFR, ALK or ROS-1 have improved significantly. ${ }^{3}$

KRAS is commonly mutated in lung cancer and especially prevalent in NSCLC. ${ }^{4,5}$ Mutations in KRAS constitutively activate its downstream signaling pathways ${ }^{6}$ such as MEK/ERK, a mitogen-activated protein kinase (MAPK) pathway, this is known to promote cancer cell survival and chemoresistance. ${ }^{7,8}$ KRAS mutations correlate significantly to poor therapeutic response in NSCLC patients. ${ }^{9-11}$ Therefore, it is essential to develop novel KRAS-targeting drugs for treating recalcitrant NSCLC patients with KRAS mutations. 
Anlotinib is a novel tyrosine kinase inhibitor (TKI) ${ }^{12}$ that targets multiple TKs including VEGFR, PDGFR, FGFR and c-Kit. It has shown potent therapeutic effects against renal cell carcinoma, thyroid cancer and osteosarcoma in preclinical and clinical trials. Wang showed that Anlotinib blocked osteosarcoma development and progression by inhibiting VEGFR2 and MET. ${ }^{13}$ Likewise, Lin and Xie reported anti-angiogenic effects of Anlotinib via suppression of VEGFR, PDGFR, FGFR and c-Kit. ${ }^{14,15}$ At present, Anlotinib is approved in China as a single drug therapy for patients with locally advanced or metastatic NSCLC who present tumor progression or recurrence after undergoing at least 2 lines of systemic chemotherapy. ${ }^{16}$ The Phase II (ALTER0302) and Phase III (ALTER0303) clinical trials conducted by Han showed that Anlotinib significantly improved the OS and PFS of patients with advanced NSCLC as a third-line treatment compared to the placebo, and was well tolerated. ${ }^{17-19} \mathrm{Si}$ also reported that Anlotinib improved the quality of life in advanced NSCLC patients, and recommended it for the third-line and subsequent treatment. ${ }^{20}$ Recently, a case report demonstrated that an advanced lung adenocarcinoma patient with KRAS mutation responded well to Anlotinib, thus indicating its suitability for recalcitrant KRAS mutant NSCLC. However, no preclinical studies so far have analyzed the effects of Anlotinib on KRAS mutant NSCLC or explored the possible molecular mechanisms. To this end, we evaluated the effects of Anlotinib on lung cancer cells harboring the KRAS mutation both in vitro and in vivo, and investigated the underlying molecular mechanisms.

\section{Materials and Methods Cell Culture}

The human NSCLC cell lines A549 and NCI-H460 respectively harboring the G12S and Q61H KRAS mutations were purchased from American Type Culture Collection (ATCC). The cells were cultured at $37^{\circ} \mathrm{C}$ in RPMI 1640 medium containing 10\% fetal bovine serum (FBS, Gibco) and $1 \%$ antibiotics (Penicillin 100U/mL, Streptomycin 100 $\mathrm{ug} / \mathrm{mL}$ ) under $5 \% \mathrm{CO}_{2}$. All experiments were conducted in the exponential growth phase.

\section{CCK8 Assay}

The A549 and NCI-H460 cells were seeded in 96-well plates at the density of $3 \times 10^{3}$ cells/well and incubated overnight. Following treatment with different concentrations
$(10,20,30$, and $40 \mu \mathrm{M})$ of Anlotinib (China Tai Tianqing Pharmaceutical Group Co. Ltd.) for 48h, the CCK-8 reagent (Dojindo, Kumamoto, Japan) was added, and the cells were incubated for another hour at $37^{\circ} \mathrm{C}$. The percentage of viable cells was determined by measuring the absorbance at $450 \mathrm{~nm}$ using a microplate reader (Bio-Rad, USA). The median inhibitory concentration ( $\mathrm{IC}_{50}$ value) was calculated using Prism 7.0 software (GraphPad Software).

\section{Colony Formation Assay}

The cells were seeded in 6-well plates at the density of 500 cells/well and incubated overnight. After culturing the cells with different concentrations of Anlotinib for 14 days, the ensuing colonies were stained with $0.1 \%$ crystal violet for $20 \mathrm{~min}$. The number of colonies (> 50 cells) were counted under a Leica DM1400B inverted microscope linked to a camera.

\section{Flow Cytometry}

The cells were seeded in 6-well culture plates at the density of $2 \times 10^{5}$ cells $/ \mathrm{mL}$ and after an overnight culture, treated with different concentrations of Anlotinib for $48 \mathrm{~h}$. After washing twice with PBS, the cells were harvested and stained with Annexin V + 7-AAD reagent (Keygen, Biotech, Nanjing, China) at room temperature for $15 \mathrm{~min}$ in the dark according to the manufacturer's protocol. The percentage of apoptotic cells was evaluated by flow cytometry (BD FACSCalibur; BD Bioscience).

\section{Wound Healing Scratch Assay}

The cells were seeded in 6-well plates and grown until confluent. The monolayer was scratched with a sterile pipette tip, and the dislodged cells were washed. Images of the "wound" region were taken at $0 \mathrm{~h}$ and $48 \mathrm{~h}$ of culture with different concentrations of Anlotinnib under a cameraequipped light microscope (Leica, Japan).

\section{Transwell Assay}

Transwell chambers $(8 \mu \mathrm{m}$ pore size; Corning Costar, USA) coated with Matrigel (BD Biosciences, San Jose, CA, USA) were placed in a 24-well culture plate. The lower chambers were filled with $600 \mu \mathrm{L}$ complete RPMI 1640 medium, and the cells were seeded in the upper chambers in $200 \mu \mathrm{L}$ serum-free medium. After culturing the cells with different concentrations of Anlotinib for 48h, the transwell chambers were transferred into a fresh plate, washed with PBS, fixed with $4 \%$ paraformaldehyde for $20 \mathrm{~min}$ and stained with $0.1 \%$ crystal violet (CV) for 
15 min. The number of invasive cells were counted under a Leica DM1400B inverted microscope linked to a camera.

\section{Western Blotting}

The suitably treated A546 and NCI-H460 cells were homogenized with RIPA lysis buffer supplemented with a protease and phosphatase inhibitor cocktail (Beyotime Biotechnology). The protein concentration of the lysates was measured using bicinchoninic acid assay (BCA). Equal amounts of protein per sample were separated by $10 \%$ SDS-PAGE and then electro-transferred to a PVDF membrane for $40 \mathrm{~min}$ at $4^{\circ} \mathrm{C}$. After blocking with $5 \%$ skimmed milk/Tris-Tween buffer saline (TBST) for $1 \mathrm{~h}$ at room temperature, the membranes were incubated overnight with the primary antibodies against ERK, p-ERK, MEK, p-MEK (1:1000, Cell Signaling Technology, CST), BAX, BCL-2 and $\beta$-actin (1:1000, Abcam, MA, USA). The membranes were then washed with TBST and incubated with horseradish peroxidase-conjugated goat antirabbit IgG for $60 \mathrm{~min}$. The positive bands were detected using an electrochemiluminescence (ECL) reagent.

\section{Xenograft Mouse Model}

The animal experiments were conducted in accordance with the guidelines of the Animal Care and Use Committee of West China Hospital of Sichuan University, and approved by the Animal Ethics Committee of Sichuan University.
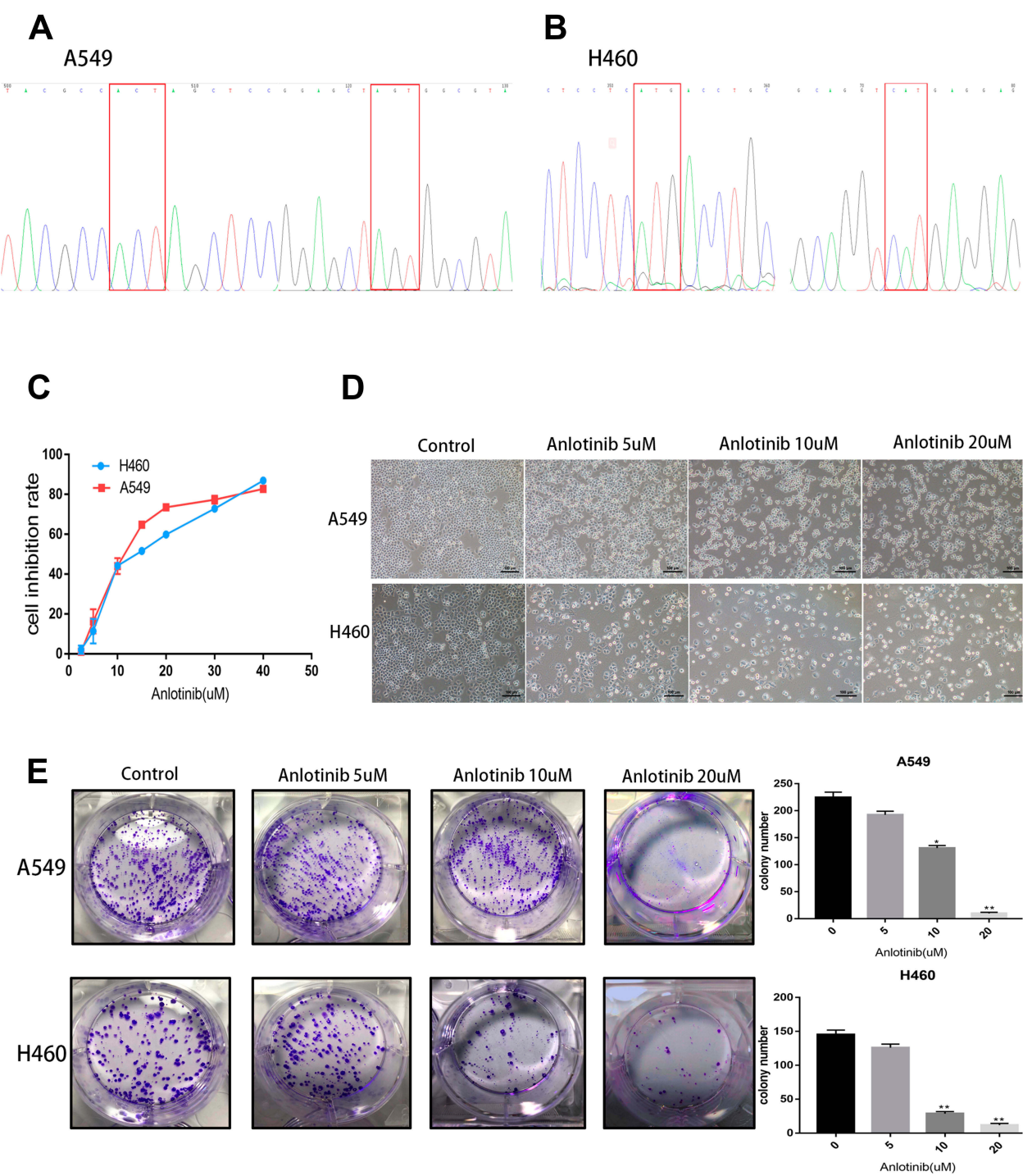

Figure I Anlotinib inhibits proliferation of KRAS mutant lung cancer cells. (A and B) KRAS mutation sites of A549 and NCI-H460 cells (GI2S and Q6IH, respectively). (C) Cell inhibition rate with Anlotinib treatment. (D) Phase contrast microphotograph images of lung cancer cells with or without Anlotinib for 48h. (E) Colony formation after Anlotinib treatment. $* P<0.05, * * P<0.01$ 
Forty female nude mice (five-weeks-old) were purchased from Beijing HFK Bioscience Co. Ltd. (Beijing, China). To establish the xenograft model, approximately $2 \times 10^{6}$ A549 or NCI-H460 cells were subcutaneously injected into the right flank of each mouse. Once the tumors grew to $50 \mathrm{~mm}^{3}-100 \mathrm{~mm}^{3}$, the mice were randomly divided into the A549 Ctrl, A549 Anlotinib, H460 Ctrl and H460 Anlotinib groups ( $\mathrm{n}=10 \mathrm{each}$ ), and injected intraperitoneally with normal saline or $5 \mathrm{mg} / \mathrm{kg}$ Anlotinib once daily for 14 days. The tumors were measured on alternate days using calipers and the volume $\left(\mathrm{mm}^{3}\right)$ was calculated as length $/ 2 \times$ width $^{2}$. Five randomly selected mice from each group were sacrificed by cervical dislocation, and the tumors were harvested, weighed and processed for immunohistochemical analysis. The remaining were observed for 70 days, and a death was recorded when a mouse died during treatment or if the tumor volume reached $1500 \mathrm{~mm}^{3}$.

\section{Immunohistochemistry (IHC)}

The freshly resected tumors were immersed in formalin and fixed overnight, embedded in paraffin, and cut into $4 \mu \mathrm{m}$-thick serial sections. After dewaxing and dehydration,

A
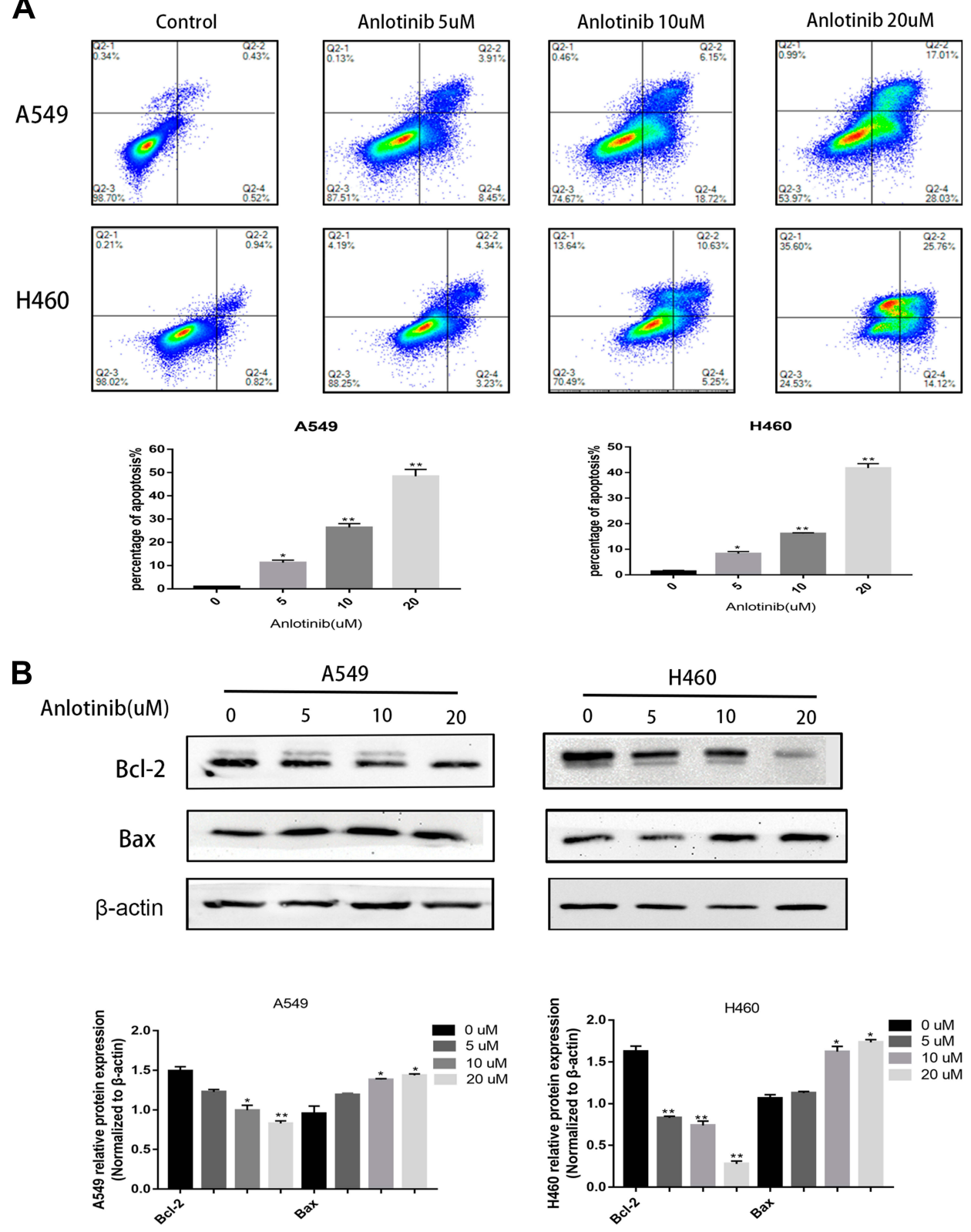

Figure 2 Anlotinib shows apoptosis induction in KRAS mutant lung cancer cells. (A) Flow cytometry detection of apoptotic cells after Anlotinib treatment. (B) Western blot analysis of $\mathrm{Bcl}-2$ and Bax level after Anlotinib treatment. $* P<0.05 * * P<0.01$. 
the sections were heated in citrate buffer for antigen retrieval. The processed sections were incubated with anti-CD31 and anti-Ki67 primary antibodies (1:1000, Abcam, MA, USA), followed by the secondary antibodies according to the manufacturer's instructions, and sealed with neutral gum. Vessel density was determined in terms of CD31 expression, and the percentage of Ki-67 positive proliferating cells was evaluated.

\section{Statistical Analysis}

Data are expressed as mean \pm standard deviation. Statistical analyses were performed using the Statistical Package for the
Social Sciences 24.0 (SPSS Inc., Chicago, IL, USA). The groups were compared using Dunnett's $t$-test, Student's $t$-test, or one-way analysis of variance with StudentNewman-Keuls ad hoc test as appropriate. $\mathrm{P}<0.05$ was considered statistically significant.

\section{Results}

\section{Anlotinib Inhibits Proliferation of KRAS Mutant Lung Cancer Cells}

The specific KRAS mutations in A549 and H460 cells were verified by sequencing. As shown in Figure 1A and B,

A
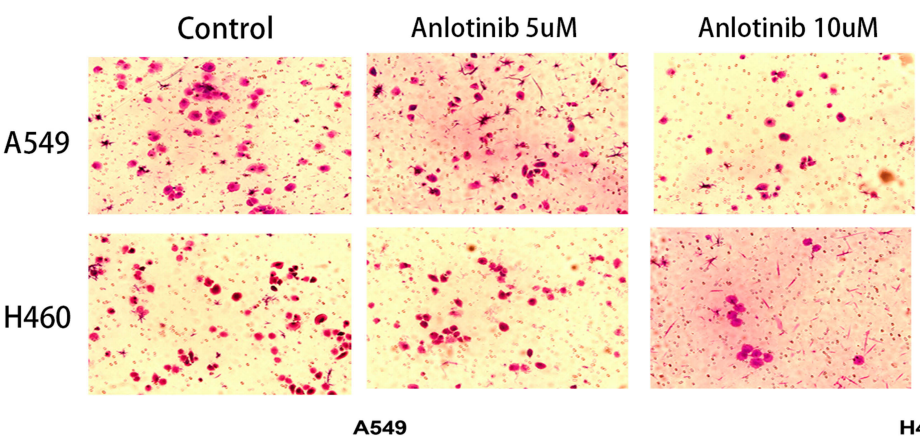

Anlotinib 20uM
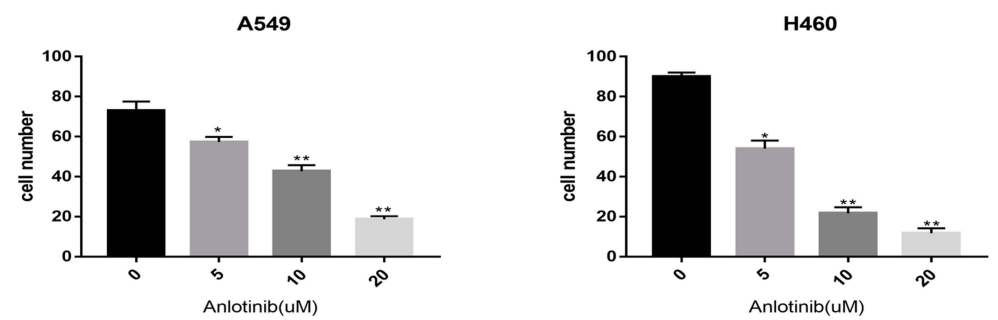

B

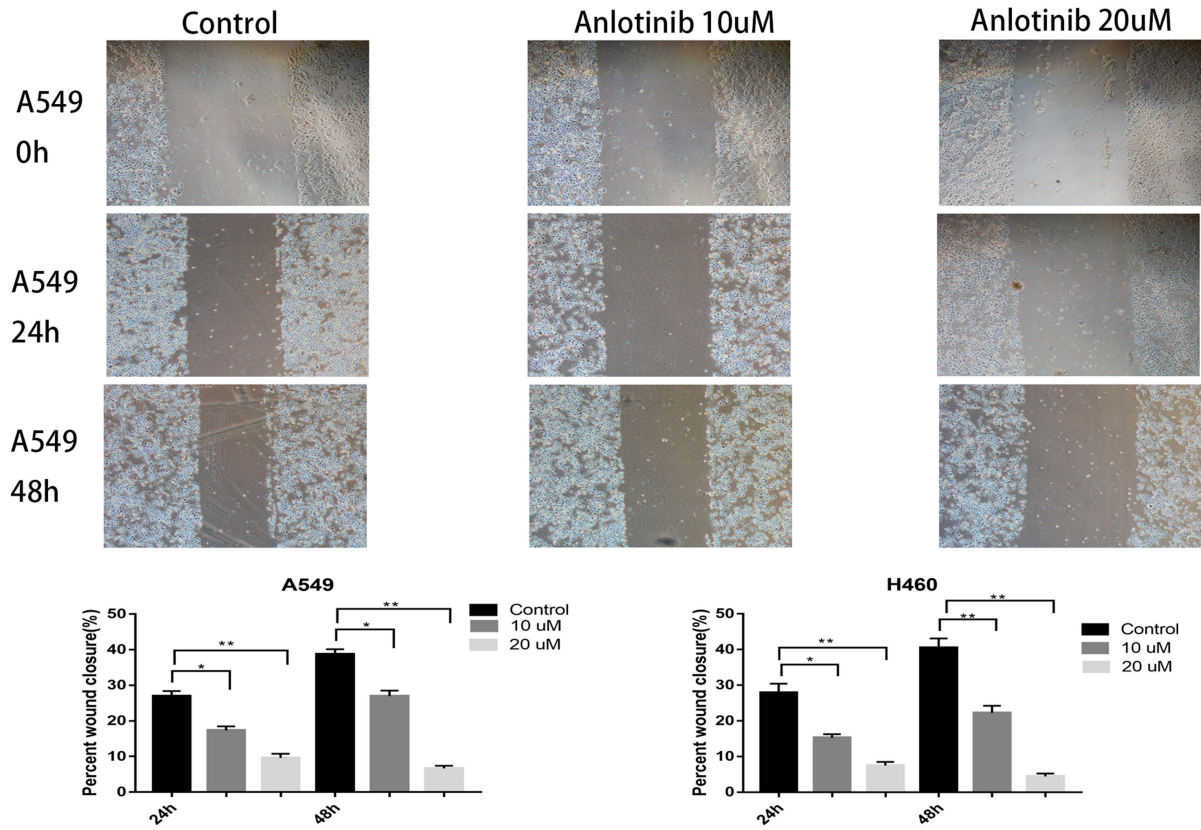

Figure 3 Anlotinib reduces metastatic potential of KRAS mutant lung cancer cells. (A) Transwell detection of invasive ability with Anlotinib treatment. (B) The Wound healing analysis of migration ability after Anlotinib treatment. $* P<0.05 * * P<0.0$ I 
KRAS was mutated at G12S and Q61H in the A549 and H460 cells respectively. Anlotinib significantly decreased the proliferation of both cell lines in a dose-dependent manner, and the respective $\mathrm{IC}_{50}$ for $\mathrm{A} 549$ and $\mathrm{H} 460$ cells were $18.84 \mu \mathrm{M}$ and $16.77 \mu \mathrm{M}$ (Figure $1 \mathrm{C}$ and D). Anlotinib treatment also markedly decreased the number of colonies formed by the A549 and H460 cells (Figure 1E). Furthermore, the proportion of apoptotic A549 cells increased from $12.36 \%$ to $45.05 \%$ and that of apoptotic H460 cells from $7.57 \%$ to $39.88 \%$ after Anlotinib treatment (Figure 2A). Consistent with this, Anlotinib significantly downregulated the levels of the anti-apoptotic BCL-2

A

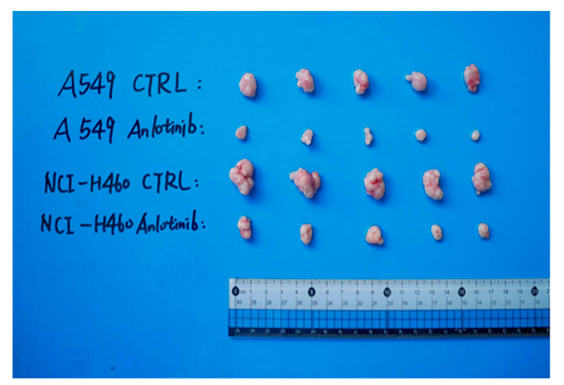

C

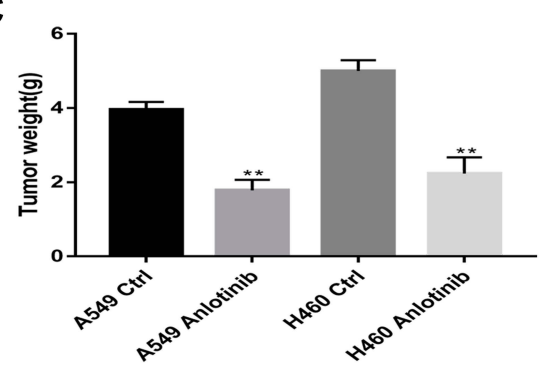

E

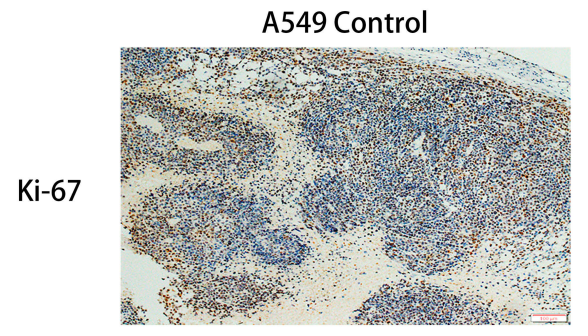

H460 Control

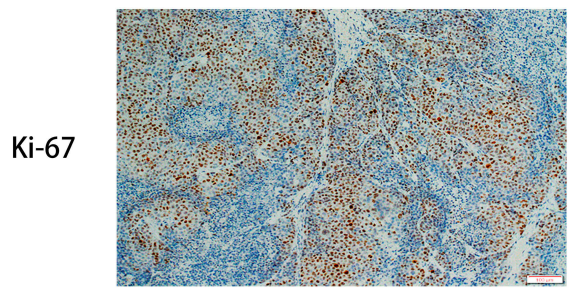

protein and upregulated the pro-apoptotic BAX in A549 and $\mathrm{H} 460$ cells (Figure 2B).

\section{Anlotinib Reduces Migration and Invasion of KRAS Mutant Lung Cancer Cells}

To determine the possible effect of Anlotinib on the metastatic potential of KRAS mutant lung cancer cells, we performed the in vitro wound healing and transwell assays. As shown in Figure 3B, Anlotinib inhibited the migratory ability of A549 and H460 cells, and also significantly decreased the number of invasive cells (Figure 3A).

\section{B}

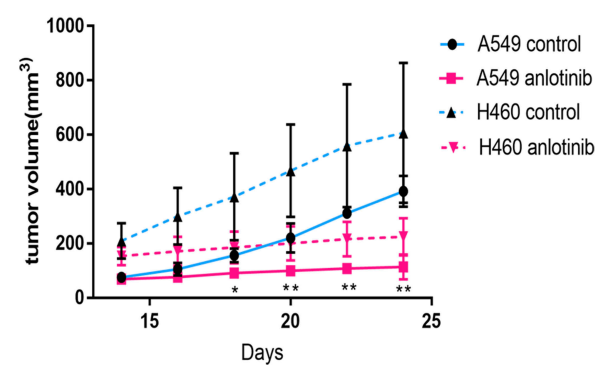

D
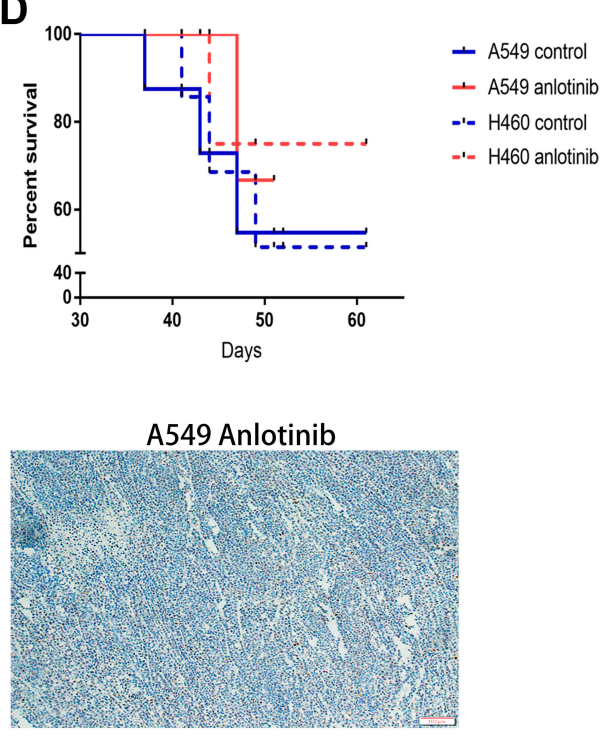

H460 Anlotinib

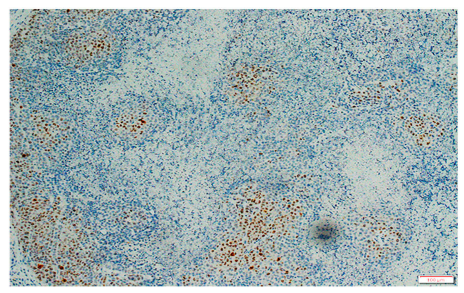

Figure 4 Anlotinib exerts anti-cancer effects in vivo. (A) Anlotinib suppresses tumor growth of $\mathrm{NCl}-\mathrm{H} 460$ and $\mathrm{A} 549$ cells bearing mice. (B) Tumor volume of $\mathrm{NCl}-\mathrm{H} 460$ and A549 cells bearing mice. (C) Tumor weight of $\mathrm{NCl}-\mathrm{H} 460$ and A549 cells bearing mice. (D) Survival time of $\mathrm{NCl}-\mathrm{H} 460$ and A549 cells bearing mice. (E) IHC detection of $\mathrm{Ki} 67$ expression in tumor tissues of $\mathrm{NCl}-\mathrm{H} 460$ and $\mathrm{A} 549$ cells bearing mice. $* * P<0.01$. Scale bar $=100$ um. 


\section{Anlotinib Suppresses Growth of KRAS Mutant Xenografts in Mice}

To further validate the anti-cancer effects of Anlotinib in vivo, we established KRAS mutant lung cancer xenografts in a mouse model. As shown in Figure $4 \mathrm{~A}$ and $\mathrm{B}$ and Supplementary Figure 1A, Anlotinib significantly suppressed the growth of KRAS mutant tumors compared to that in the untreated controls, without affecting the body weight of mice. Consistent with this, the post-mortem tumor weight was significantly lower in the Anlotinib-treated versus the control group (Figure 4C). In addition, Anlotinib significantly decreased the expression of $\mathrm{Ki67}$ in the tumor tissues
(Figure 4E), and prolonged the survival of the tumor-bearing mice (Figure 4D). We also detected the extent of neoangiogenesis in the tumor tissues by CD31 immunostaining, and observed a significant reduction in CD31+ vessel density in the treated versus the untreated group (Supplementary Figure 1B), indicating that Anlotinib also exerted its anticancer effects by suppressing angiogenesis.

\section{Anlotinib Attenuates MEK/ERK Pathway in KRAS Mutant Lung Cancer Cells}

To explore the possible molecular mechanism of Anlotinib action, we analyzed the expression levels of the ERK and
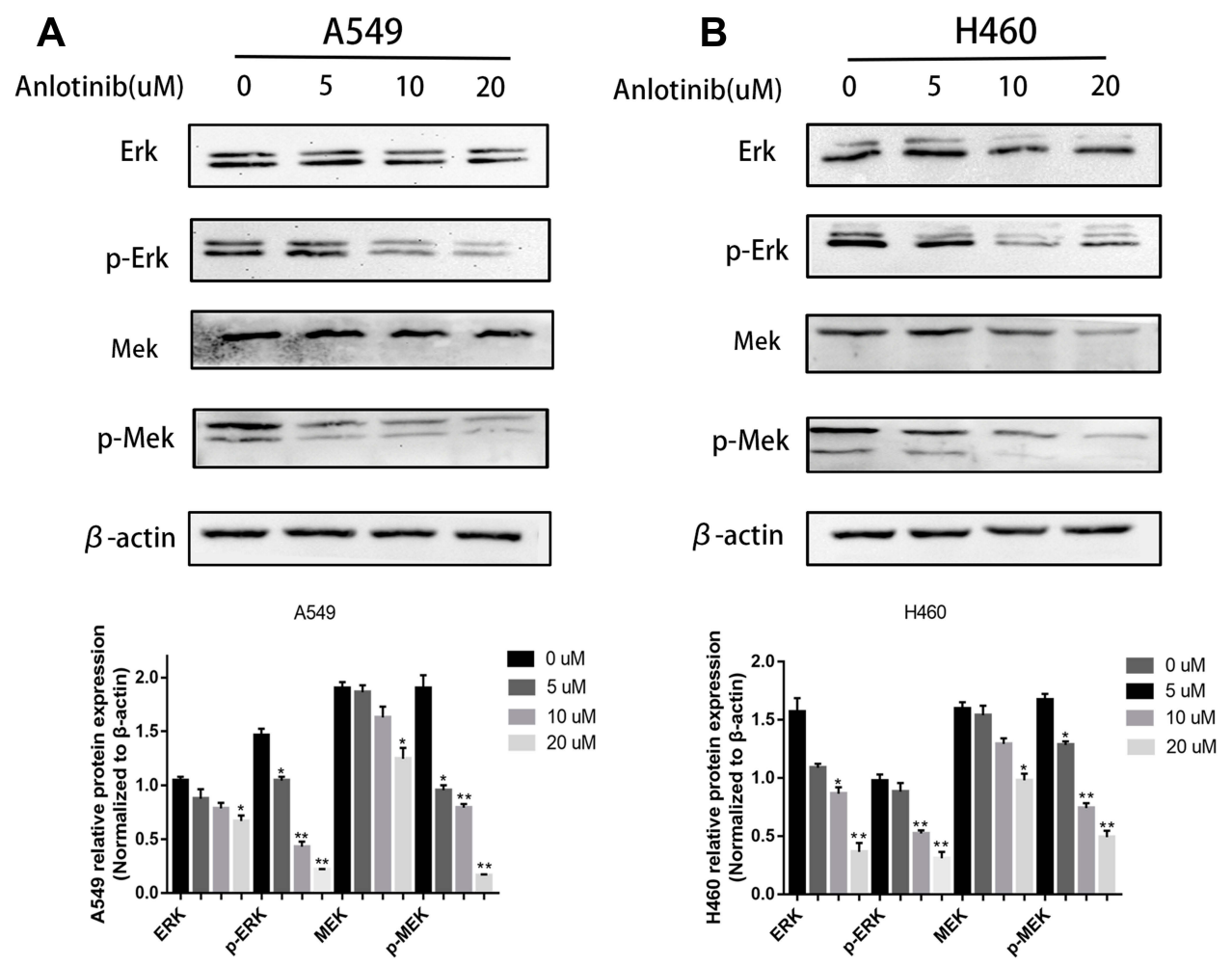

C

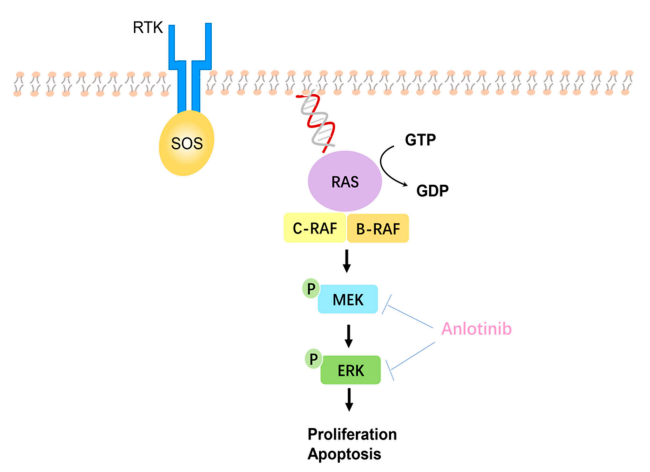

Figure 5 Anlotinib attenuates MEK/ERK pathway in KRAS mutant lung cancer cells. (A) Western blot analysis of ERK, P-ERK, MEK, p-MEK in A549 cells after Anlotinib treatment. (B) Western blot analysis of ERK, p-ERK, MEK, p-MEK in NCl-H460 cells after Anlotinib treatment. (C) MAPK signaling pathway in KRAS mutant cancers. $* P<0.05, * * P<0.01$. 
MEK. Anlotinib downregulated both ERK and MEK, and also significantly decreased the levels of p-ERK and $\mathrm{p}-\mathrm{MEK}$ in a concentration-dependent manner (Figure 5A and B). Thus, Anlotinib inhibits the growth of KRAS mutant cells by blocking MAPK signaling. The putative mechanism is outlined in Figure $5 \mathrm{C}$.

\section{Discussion}

Lung cancer is associated with high incidence and mortality, and the KRAS mutations, especially in NSCLC patients, render the tumors recalcitrant to treatment. ${ }^{21-23}$ Anlotinib is an orally-administered multi-target TKI (RTKs). In the present study, we found that Anlotinib inhibited proliferation of KRAS mutant lung cancer cells in vitro and in vivo, and prolonged the survival of tumorbearing mice, which is consistent with a recent clinical case report of a lung adenocarcinoma patient. ${ }^{11}$ A previous study showed that Anlotinib inhibited hepatocellular carcinoma cells via apoptosis induction. ${ }^{24}$ In this study as well, Anlotinib significantly induced apoptosis in the KRAS mutant lung cancer cells by downregulating the survival factor BCL-2 and upregulating the pro-apoptotic factor $\mathrm{BAX}^{25,26}$ in a dose-dependent manner.

Metastasis is an indicator of poor prognosis in lung cancer patients, and decreasing the metastatic potential of lung cancer cells significantly prolonged the survival of xenograft-bearing mice. ${ }^{27,28}$ Recently, Liu Z reported significantly higher risk of metastasis in the KRAS mutant versus wild-type lung cancer patients. ${ }^{29}$ Consistent with this, Anlotinib markedly decreased the migratory and invasive abilities of KRAS mutant lung cancer cells.

The MEK/ERK signal is one of the MAPK pathways which can regulate multiple cellular processes, including proliferation, apoptosis and chemoresistance. ${ }^{30,31}$ Recently, Yen and Ji D reported that KRAS mutation in lung cancer cells increased the levels of phosphorylated MEK and ERK, and then promoted proliferation and suppressed apoptosis in lung cancer cells. ${ }^{6,32,33} \mathrm{MEK} / \mathrm{ERK}$ signaling plays an important role in KRAS mutant lung cancer cells. And it was found that the phosphorylation of MEK/ERK could be repressed by Anlotinib in this study.

\section{Conclusions}

Anlotinib can be included as part of the first-line treatment of KRAS mutant NSCLC patients, and needs to be validated by further functional and clinical studies.

\section{Acknowledgments}

This research was supported by the National Natural Science Foundation of China (no.81802512), and the Project of Science and Technology Department of Sichuan Province (no.2018FZ0115).

\section{Author Contributions}

All authors contributed to data analysis, drafting or revising the article, give final approval of version to be published, and agree to be accountable for all aspects of the work.

\section{Disclosure}

The authors declare that there is no conflict of interest.

\section{References}

1. Duruisseaux M, Esteller M. Lung cancer epigenetics: from knowledge to applications. Semin Cancer Biol. 2018;51:116-128. doi:10.1016/j.semcancer.2017.09.005

2. Goulart BH, Martins RG, Lynch TJ. Twenty-two years of phase III trials for patients with advanced non-small-cell lung cancer: sobering results. J Clin Oncol. 2001;19(20):4089. doi:10.1200/JCO.2001.19.10.2674

3. Clark JW, Longo DL. Recent progress in systemic treatment for lung cancer. Curr Opin Pulm Med. 2018;24(4):355-366. doi:10.1097/ MCP.0000000000000493

4. Korpanty GJ, Graham DM, Vincent MD, Leighl NB. Biomarkers that currently affect clinical practice in lung cancer: EGFR, ALK, MET, ROS-1, and KRAS. Front Oncol. 2014;4:204. doi:10.3389/fonc.2014. 00204

5. Morris TA, Khoo C, Solomon BJ. Targeting ROS1 rearrangements in non-small cell lung cancer: crizotinib and newer generation tyrosine kinase inhibitors. Drugs. 2019;79(12):1277-1286. doi:10.1007/ s40265-019-01164-3

6. Yen I, Shanahan F, Merchant M, et al. Pharmacological Induction of RAS-GTP confers RAF inhibitor sensitivity in KRAS mutant tumors. Cancer Cell. 2018;34(4):611-625.e617. doi:10.1016/j.ccell.2018.09.002

7. Parameswaran N, Bartel CA, Hernandez-Sanchez W, et al. A FAM83A positive feed-back loop drives survival and tumorigenicity of pancreatic ductal adenocarcinomas. Sci Rep. 2019;9(1):13396. doi:10.1038/s41598-019-49475-5

8. Bardia A, Gounder M, Rodon J, et al. Phase Ib study of combination therapy with MEK inhibitor binimetinib and phosphatidylinositol 3-kinase inhibitor buparlisib in patients with advanced solid tumors with RAS/RAF alterations. Oncologist. 2020;25(1):e160.

9. Ito M, Codony-Servat C, Codony-Servat J, et al. Targeting PKCiota-PAK1 signaling pathways in EGFR and KRAS mutant adenocarcinoma and lung squamous cell carcinoma. Cell Commun Signal. 2019;17(1):137. doi:10.1186/s12964-019-0446-z

10. Aredo JV, Padda SK. Management of KRAS-mutant non-small cell lung cancer in the era of precision medicine. Curr Treat Options Oncol. 2018;19(8):43. doi:10.1007/s11864-018-0557-6

11. Su Y, Meng Z, Xu X, et al. A case report of advanced lung adenocarcinoma harboring KRAS mutation treated with anlotinib. Zhongguo Fei Ai Za Zhi. 2018;21(5):428-430. doi:10.3779/j.issn.1009-3419.2018.05.13

12. Shen G, Zheng F, Ren D, et al. Anlotinib: a novel multi-targeting tyrosine kinase inhibitor in clinical development. $J$ Hematol Oncol. 2018;11(1):120. doi:10.1186/s13045-018-0664-7

13. Wang G, Sun M, Jiang Y, et al. Anlotinib, a novel small molecular tyrosine kinase inhibitor, suppresses growth and metastasis via dual blockade of VEGFR2 and MET in osteosarcoma. Int $J$ Cancer. 2019;145(4):979-993. doi:10.1002/ijc.32180 
14. Xie C, Wan X, Quan H, et al. Preclinical characterization of anlotinib, a highly potent and selective vascular endothelial growth factor receptor-2 inhibitor. Cancer Sci. 2018;109(4):1207-1219. doi:10.1111/cas.13536

15. Lin B, Song X, Yang D, Bai D, Yao Y, Lu N. Anlotinib inhibits angiogenesis via suppressing the activation of VEGFR2, PDGFR $\beta$ and FGFR1. Gene. 2018;654:77-86. doi:10.1016/j.gene.2018.02.026

16. Syed YY. Correction to: anlotinib: first global approval. Drugs. 2018;78(12):1287. doi:10.1007/s40265-018-0961-Z

17. Han B, Li K, Zhao Y, et al. Anlotinib as a third-line therapy in patients with refractory advanced non-small-cell lung cancer: a multicentre, randomised phase II trial (ALTER0302). Br J Cancer. 2018;118 (5):654-661. doi:10.1038/bjc.2017.478

18. Han B, Li K, Wang Q, et al. Effect of anlotinib as a third-line or further treatment on overall survival of patients with advanced NonSmall cell lung cancer. JAMA oncol. 2018;4(11):1569-1575. doi:10.1001/jamaoncol.2018.3039

19. Zhou M, Chen X, Zhang H, et al. China National Medical Products Administration approval summary: anlotinib for the treatment of advanced non-small cell lung cancer after two lines of chemotherapy. Cancer Commun (Lond). 2019;39(1):36.

20. Si X, Zhang L, Wang H, et al. Quality of life results from a randomized, double-blinded, placebo-controlled, multi-center phase III trial of anlotinib in patients with advanced non-small cell lung cancer. Lung Cancer. 2018;122:32-37. doi:10.1016/j.lungcan.2018.05.013

21. Jones GG, Del Rio IB, Sari S, et al. SHOC2 phosphatase-dependent RAF dimerization mediates resistance to MEK inhibition in RAS-mutant cancers. Nat Commun. 2019;10(1):2532. doi:10.1038/ s41467-019-10367-x

22. Wang $\mathrm{H}, \mathrm{Lv} \mathrm{Q}, \mathrm{Xu} \mathrm{Y}$, et al. An integrative pharmacogenomics analysis identifies therapeutic targets in KRAS-mutant lung cancer. EBioMedicine. 2019;49:106-117. doi:10.1016/j.ebiom.2019.10.012

23. Johnson DB, McDonnell WJ, Gonzalez-Ericsson PI, et al. A case report of clonal EBV-like memory $\mathrm{CD} 4(+) \mathrm{T}$ cell activation in fatal checkpoint inhibitor-induced encephalitis. Nat Med. 2019;25 (8):1243-1250. doi:10.1038/s41591-019-0523-2

24. He C, Wu T, Hao Y. Anlotinib induces hepatocellular carcinoma apoptosis and inhibits proliferation via Erk and Akt pathway. Biochem Biophys Res Commun. 2018;503(4):3093-3099. doi:10.1016/j.bbrc.2018.08.098
25. Adams CM, Clark-Garvey S, Porcu P, Eischen CM. Targeting the Bcl-2 Family in B cell lymphoma. Front Oncol. 2018;8:636. doi:10.3389/fonc.2018.00636

26. Yan $\mathrm{X}, \mathrm{Gu} \mathrm{Y}$, Wang $\mathrm{C}$, et al. Unbalanced expression of membrane-bound and soluble inducible costimulator and programmed cell death 1 in patients with myasthenia gravis. Clin Immunol. 2019;207:68-78. doi:10.1016/j.clim.2019.07.011

27. Psimaras D, Velasco R, Birzu C, et al. Immune checkpoint inhibitors-induced neuromuscular toxicity: from pathogenesis to treatment. J Peripher Nerv Syst. 2019;24(Suppl 2):S74-S85. doi:10.1111/jns.12339

28. Bernhardt D, Konig L, Aufderstrasse S, et al. Generation of a new disease-specific prognostic score for patients with brain metastases from small-cell lung cancer treated with whole brain radiotherapy (BMS-Score) and validation of two other indices. Clin Lung Cancer. 2018;19(4):340-345. doi:10.1016/j.cllc.2017.12.004

29. Liu Z, Liang H, Lin J, et al. The incidence of lymph node metastasis in patients with different oncogenic driver mutations among T1 non-small-cell lung cancer. Lung Cancer. 2019;134:218-224.6. doi:10.1016/j.lungcan.2019.06.026

30. Arscott WT, Zhu S, Plastaras JP, Maity A, Alonso-Basanta M, Jones J. Acute neurologic toxicity of palliative radiotherapy for brain metastases in patients receiving immune checkpoint blockade. Neurooncol Pract. 2019;6(4):297-304. doi:10.1093/nop/npy042

31. Mencel J, Gargett T, Karanth N, Pokorny A, Brown MP, Charakidis M. Thymic hyperplasia following double immune checkpoint inhibitor therapy in two patients with stage IV melanoma. Asia Pac J Clin Oncol. 2019;15(6):383-386. doi:10.1111/ajco.13233

32. Ji D, Zhang L, Zhu Q, Bai Y, Wu Y, Xu Y. Discovery of potent, orally bioavailable ERK1/2 inhibitors with isoindolin-1-one structure by structure-based drug design. Eur J Med Chem. 2019;164:334-341. doi:10.1016/j.ejmech.2018.12.040

33. Lv J, Zhang F, Zhai C, Wang G, Qu Y. Bag-1 silence sensitizes non-small cell lung cancer cells to cisplatin through multiple gene pathways. Onco Targets Ther. 2019;12:8977-8989. doi:10.2147/OTT. S218182

\section{Publish your work in this journal}

Cancer Management and Research is an international, peer-reviewed open access journal focusing on cancer research and the optimal use of preventative and integrated treatment interventions to achieve improved outcomes, enhanced survival and quality of life for the cancer patient.
The manuscript management system is completely online and includes a very quick and fair peer-review system, which is all easy to use. Visit http://www.dovepress.com/testimonials.php to read real quotes from published authors. 